\title{
Indagine conoscitiva: la valutazione e la gestione del paziente portatore di lesioni cutanee \\ Learning survey: assessment and management of the patient with wounds
}

\author{
Elisabetta Sardone, ${ }^{1}$ Elena Toma ${ }^{2}$ \\ ${ }^{1}$ ASL Roma 2; ${ }^{2}$ Libera professionista, Roma, Italia
}

\begin{abstract}
RIASSUNTO
L'obiettivo è stato di esplorare come realmente viene svolta la gestione e la valutazione del paziente portatore di lesioni cutanee (LC) nel territorio italiano individuando: i metodi utilizzati per la valutazione; se e come è attualmente documentato il percorso assistenziale; la capacità operative e livello formativo degli operatori coinvolti; quanto è percepita l'esigenza dell'operatore di avere uno strumento guida dedicato. Lo studio è stato eseguito attraverso un'indagine conoscitiva rivolta alla professione infermieristica, sviluppata con l'ausilio di un questionario dedicato validato, con distribuzione cartacea e online (1200 questionari) su un campione d'indagine di 726 questionari. Il profilo della popolazione coinvolta è: $71 \%$ di genere femminile, 54\% di età superiore ai 40 anni, $84 \%$ infermiere, $16 \%$ infermiere Wound Care Specialist (WS), 63\% lavora in struttura ospedaliera, 13\% effettua 21-100 medicazioni ogni 15 gg. Riguardo l'utilizzo della documentazione emerge che: $53 \%$ dei professionisti non utilizza documentazione standardizzata, ma come strumenti prevalenti la cartella clinica ed il diario di consegna; 18,60\% non documenta l'attività e non usa scale validate. Risulta inoltre che: $65 \%$ degli operatori non valuta complessivamente la lesione e $78 \%$ non valuta il letto della lesione, $94 \%$ non valuta lo stato nutrizionale del paziente, 93\% esprime l'esigenza di avere uno strumento che guidi e documenti l'attività di valutazione e gestione della LC. Solo $59 \%$ degli operatori conosce i servizi territoriali dedicati alla cura delle LC e il 70\% è al corrente dell'esistenza del Master Universitario di I Livello. È quindi risultata radicata la cattiva pratica di non effettuare la valutazione del rischio, la valutazione della lesione e dello stato nutrizionale. Alla luce dei dati emersi, la situazione riscontrata è incompatibile con un sistema sanitario efficace e sono compromesse la qualità e appropriatezza degli interventi per la cura delle persone affette da lesioni cutanee a tutti i livelli.
\end{abstract}

\section{ABSTRACT}

The aim was to explore how the management and assessment of the patient with skin lesions (SL) is actually carried out in the Italian territory by identifying: the methods used for the evaluation; whether and how the care pathway is currently documented; the ability and training level of the operators involved; how much the operator perceives the need of having a specific guide tool. The study was carried out through a consultation focused on the nursing profession, developed with the help of a dedicated and validated questionnaire, with printed and online distribution (1200 questionnaires) on a survey sample of 726 questionnaires. The profile of the involved population is: 71\% female gender, 54\% over forty, 84\% nurse, 16\% Wound Care Specialist (WS) nurse, 63\% working in hospital, 13\% making 21-100 dressings every 15 days. As regards the use of scientific documentations, it emerges that: $53 \%$ of professionals does not use standardized documentation, but, as prevalent tools, they use medical records and delivery diary; $18.60 \%$ does not document the activities and does not use rating scales. It also appears that: $65 \%$ does not evaluate the lesion and $78 \%$ does not evaluate the wound bed, 94\% does not evaluate the nutritional status of the patient, 93\% expressed the requirement to have a tool to guide and document the evaluation and management of SL. Only 59\% knows the territorial services dedicated to the care of the SL and the $70 \%$ is aware of the existence of the University Master's degree. The bad practice of not carrying out the risk assessment, the assessment of the lesion and the nutritional status has therefore been rooted. In the light of the emerged data, the situation found is incompatible with an effective health system and the quality and appropriateness of interventions for the treatment of people with skin lesions at all levels are compromised.

Ricevuto per la pubblicazione: 16 settembre 2017

Revisione ricevuta: 13 marzo 2018.

Accettato per la pubblicazione: 30 marzo 2018.

This work is licensed under a Creative Commons Attribution NonCommercial 4.0 License (CC BY-NC 4.0).

(C) Copyright E. Sardone e E. Toma, 2018

Licensee PAGEPress, Italy

Italian Journal of Wound Care 2018; 2(1):22-33

doi:10.4081/ijwc.2018.16

\section{INTRODUZIONE}

Il 07.07.2015 si è costituito un Gruppo di Lavoro con la partecipazione di AIUC Lazio, IPASVI Roma e Ordine dei Farmacisti Roma, con l'obiettivo dell'individuazione della rete assistenziale regionale e attuazione di Percorsi 
Diagnostico-Terapeutici integrati Ospedale-Territorio per la Prevenzione e Gestione delle lesioni cutanee nella regione Lazio, da sottoporre alle autorità politiche della regione Lazio, forte del contributo delle massime espressioni scientifiche e professionali attrici.

\section{MATERIALI E METODI}

Allo scopo d'individuare un percorso di prevenzione e di trattamento efficace, con il massimo riguardo all'appropriatezza operativa e organizzativa, in ottica di spending review, si è valutato necessario rilevare: i) le criticità attuali; ii) il livello di formazione degli operatori; iii) la disponibilità di personale con formazione specifica (Wound Care Specialist). Si è dunque deciso di procedere, in via preliminare, all'accertamento dello stato dell'arte mediante un'indagine conoscitiva a mezzo di un questionario dedicato, con scelte dicotomiche, validato (coerenza interna, di concordanze delle forme verbali scelte, attendibilità dei dati al reset), secondo Formula Kuder e Richardson: una domanda corretta punteggio 1 e una errata punteggio 0 . La statistica test è:

$$
\rho_{K R 20}=\frac{k}{k-1}\left(1-\frac{\sum_{j=1}^{k} p_{j} q_{j}}{\sigma^{2}}\right)
$$

Dove: $\mathrm{k}=$ numero di domande; $\mathrm{p} \mathrm{j}=$ numero di persone del campione che hanno risposto domanda $\mathrm{j}$ correttamente; $\mathrm{q} j=$ numero di persone del campione che non rispondono alla domanda $\mathrm{j}$ correttamente; $\sigma 2=$ varianza dei punteggi totali di tutte le persone che prendono il test=VARP (R1) in cui R1=array contenente i punteggi totali di tutte le persone che prendono il test.

I valori compresi tra 0 e 1 . Un valore elevato indica affidabilità, mentre un valore troppo elevato (superiore a 0.90) indica un test omogeneo, composto da: i) una parte dedicata all'anagrafica dell'intervistato; ii) uno spazio per suggerimenti; iii) 11 domande di cui 6 a risposta chiusa, 5 a risposta aperta. ${ }^{1}$

Realizzata per la pubblicazione online nei siti IPASVI Lazio e AIUC, e distribuita agli eventi IPASVI e AIUC e nei corsi residenziali con patrocinio IPASVI Roma.

L'obiettivo è conoscere le scelte operative degli infermieri in funzione del lavoro svolto a favore delle persone affette da lesioni cutanee. La cura delle lesioni cutanee negli ultimi anni ha assunto un grande peso economico nel sistema sanitario nazionale: un adeguato background permette di adottare un metodo di lavoro secondo evidence best practice $(\mathrm{EPB})^{2,3}$ per la prevenzione della lesione cutanea nei soggetti a rischio. ${ }^{4-6}$ La formazione degli operatori determina la differenza tra una scelta operativa corretta e una inappropriata, e trasforma una lesione (di qualsiasi eziologia) da acuta in una cronica. I servizi de- dicati dimostrano negli anni di migliorare la qualità di vita delle persone affette da lesione in età lavorativa, sia per quelle affette da comorbilità ${ }^{7,8}$ sia nell'assistenza domiciliare, e di realizzare un diretto vantaggio economico in termine di giorni di malattia o di prestazioni erogate.

L'indagine si prefigge l'analisi del processo adottato per la valutazione della persona affetta da lesione cutanea, il bisogno metodologico, formativo ${ }^{9}$ espresso dagli infermieri per la cura della lesione, e di esplorare il metodo di lavoro adottato in vari ambienti ed esperienze professionali, in particolare: i) l'attuale percorso gestionale del paziente portatore di lesioni cutanee; ii) se, e come, è documentata l'assistenza al paziente portatore di lesioni; iii) le capacità operative e il livello formativo degli operatori coinvolti; iv) l'esigenza percepita di utilizzare uno strumento dedicato in grado di guidare e facilitare la valutazione globale del paziente al fine di migliorare la qualità dell'assistenza.

\section{RISULTATI}

Questionari distribuiti: n. 1200, ritirati n. 900, compilati online n. 128. Dedicano l'attività a portatori di lesioni cutanee n. 650. Questionari di valida compilazione (che hanno risposto a tutte le domande): n. 600. Eliminati, incompleti o incongrui: n. 250.

Totale campione d'indagine (infermieri che hanno dichiarato di erogare assistenza a pazienti portatori di lesioni cutanee e hanno risposto a tutte le domande): n. 726.

\section{Analisi dei dati}

\section{Profilo degli infermeri coinvolti nell'indagine}

Genere: in coerenza con il dato nazionale italiano della divisione per genere degli infermieri, anche nel campione analizzato la percentuale di donne (71\%) è nettamente superiore rispetto a quella degli uomini (29\%), compatibile con la percentuale nazionale che è del 22,3\%. (Figura 1A).

Età: la distribuzione per età è proporzionalmente coerente con il dato nazionale, la maggior parte della popolazione attiva è rappresentata dalla classe maggiore di anni (54\%), e circa rispettivamente per un quarto e un terzo dalla classe di età compresa tra aa. 31-40 (27\%), e tra aa. 23-30 la frazione inferiore (19\%) (Figura 1B).

Posizione funzionale: il campione è costituito nella maggior parte da infermieri generalisti (89\%). La presenza di Wound Care Specialist (WS=coloro che hanno conseguito un Master di I livello in Wound Care) è 1'11\% dell'intera popolazione. Il dato sostanzialmente riflette lo stato generale in quanto gli operatori con specializzazione universitaria post-laurea sono generalmente pochi. ${ }^{10}$ Attualmente non abbiamo a disposizione un dato nazionale sull'entità degli infermieri Specialist (Figura 1C). 
Sede di lavoro: il campione d'indagine è costituito da un totale di n. 726 infermieri; il campione maggiormente rappresentato è quello laziale (65,79\%) (Figura 1D).

Distribuzione territoriale: il campione proviene prevalentemente dalle regioni Lazio e Puglia in funzione degli eventi più significativi in occasione dei quali è stato distribuito il questionario; in piccoli numeri sono rappresentate tutte le province italiane (Tabella 1).

È stata richiesta la sede di lavoro per valutare se essa sia un parametro influente sui modi e sulla qualità del lavoro svolto; se ne osserva la seguente distribuzione (Figura 1D): i) $16 \%$ solo in territorio; $21 \%$ struttura e territorio; iii) $63 \%$ solo in struttura. Risultano prevalenti gli operatori che lavorano solo in struttura.

Esperienza lavorativa: nell'ipotesi che l'esperienza lavorativa migliori la qualità del lavoro svolto è stato chiesto da quanto tempo è svolta l'attività, con 4 modalità di risposta (Figura 2A) e il numero di pazienti medicati nei ultimi $15 \mathrm{gg}$. di attività precedenti alla data di compilazione del questionario (Figura 2B). Si evince dunque che rispetto all'esperienza lavorativa espressa in anni, il campione è diviso come segue: i) $1 \%$ nessuna esperienza; ii) $12 \%<$ di 2 aa.; iii) $15 \%$ da 2 a 5 aa.; iv) $72 \%>$ di 5 aa. La parte più corposa è rappresentata da operatori con un'esperienza maggiore di 5 aa., ma è anche il numero maggiore di infermieri intervistati.

Numero di pazienti trattati in $15 \mathrm{gg}$. Lavorativi: sono state prese in considerazione 3 categorie (Figura 2B): i) $0-5$ pazienti $49 \%$; ii) $6-20$ pazienti $38 \%$; iii) $21-100$ pazienti $13 \%$. La categoria $0-5$ considera operatori che seguono pazienti portatori di lesioni solo occasionalmente, quella 21-100 considera operatori che svolgono attività in un servizio wound care-dedicato, e che dunque dovrebbero avere una formazione adeguata; in ulteriore dettaglio (Tabella 2) si evidenzia che il 51\% degli infermieri tratta più di 5 pazienti in $15 \mathrm{gg}$. e il $25 \%$ tratta più di 10 pazienti.

Riassumendo i dati dell'anagrafica e quelli dell'esperienza lavorativa degli operatori, si ha un campione composto da $29 \%$ uomini e $73 \%$ donne con una copertura disomogenea a livello nazionale (più rappresentate le regioni Lazio e Puglia), con preponderanza di privi di una formazione universitaria post-base specifica (11\% WS), impegnati maggiormente in struttura (63\%), dei quali il $72 \%$ con un'esperienza di lavoro $>5$ aa.

\section{Eziologia delle lesioni trattate}

Il tipo di lesione più frequentemente trattata risulta essere la lesione da pressione seguita dalla vascolare e dalla diabetica (Figura 2C). L'istogramma è calcolato sui dati assoluti, rispecchia l'andamento nazionale di prevalenza ${ }^{2}$ (Figura 3) per tipo di lesioni trattate in ambiente sia nosocomiale sia domiciliare: le lesioni da pressione e quelle vascolari assorbono rispettivamente dal $25 \%$ al $20 \%$ delle
A) GENERE

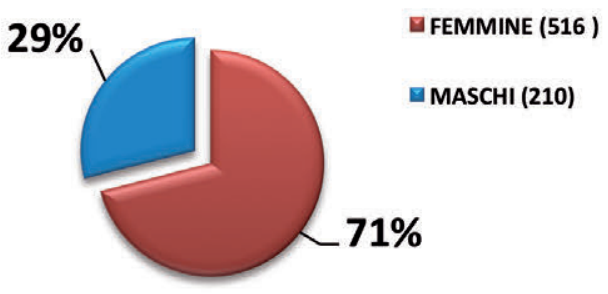

B)

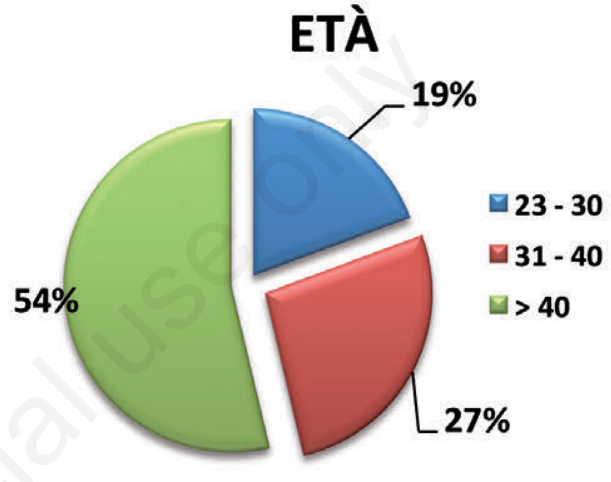

\section{C) POSIZIONE FUNZIONALE}

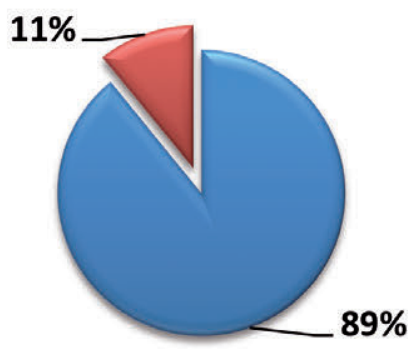

DINFERMIERE

G INFERMIERE WOUND CARE SPECIALIST

\section{D) SEDE DI LAVORO}

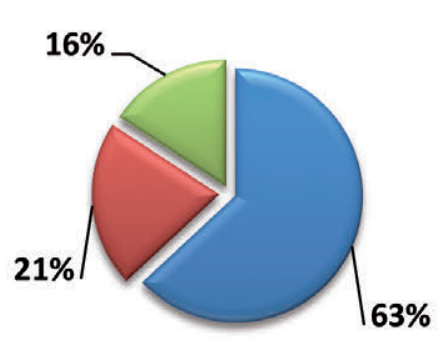

$\square$ SOLO IN STRUTTURA $\square$ STRUTTURA E TERRITORIO

Figura 1. A) Genere; B) Età; C) Posizione funzionale; D) Sede di lavoro. 
attività dedicate all'assistenza. Il dato, influenzato dalla distribuzione del questionario avvenuta presso sedi congressuali dedicate al wound care, merita specifico approfondimento.

A) ESPERIENZA LAVORATIVA

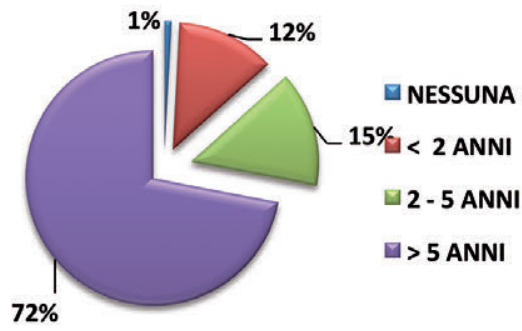

B) NUMERO DI PAZIENTI TRATTATI

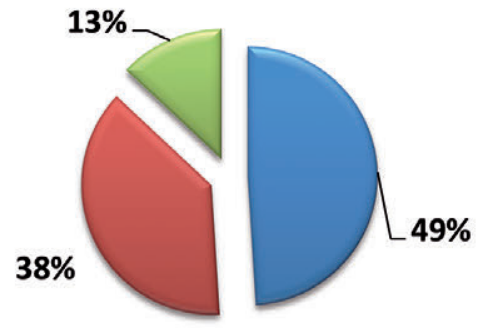

0-5 PZ.

6-20 PZ.

21-100 PZ.

C) ${ }^{3 \%}$ LESIONI TRATTATE

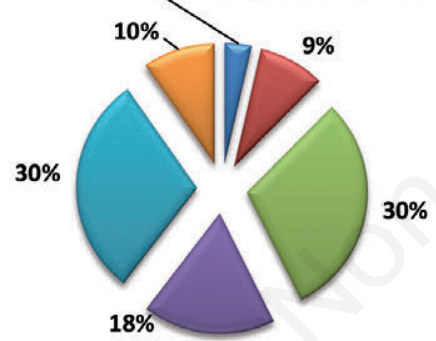

IINDETERMINATA

$\square$ TRAUMATICA

$\square$ DAPRESSIONE

$\square$ DIABETICA

$\square$ VASCOLARE

$\square$ ALTRO

Figura 2. A) Anni di esperienza lavorativa; B) Numero dei pazienti trattati negli ultimi 15 giorni; C) Eziologia delle lesioni trattate.

\section{Strumenti adottati per la valutazione del paziente affetto da lesione}

Utilizzo della documentazione: la seconda parte dell'indagine è stata dedicata all'utilizzo della documentazione e a quali criteri e indici sono prevalentemente scelti per valutazione del paziente portatore di lesione.

Documentazione standardizzata: sono stati richiesti dettagli sulla documentazione utilizzata, e se è o meno standardizzata (Figura 4A); si evince che sul totale campione il 53\% non utilizza documentazione standardizzata vs $47 \%$ che ha risposto di utilizzarla.

\section{Tipologia di documentazione utilizzata dedicata ai pazienti portatori di lesioni}

Il campione utilizza (Figura 4B): i) 38,02\% cartella; ii) $33,20 \%$ diario di consegna; iii) $10,19 \%$ appunti personali; iv) $18,60 \%$ non documenta. Il $71,22 \%$ del campione rivela di utilizzare la cartella e il diario di consegna, comunque coerente con la risposta precedente. Si rileva dunque che quasi il 30\% non usa documentazione standardizzata; tale carenza può essere attribuita anche all'inappropriata organizzazione del lavoro e all'insufficiente qualità della comunicazione tra operatori: il dato richiede approfondimento.

\section{Valutazione del paziente}

Il paziente portatore di lesioni cutanee si presenta spesso complesso e con comorbidità. ${ }^{5,6}$ In presa in carico

Tabella 2. Ulteriore dettaglio: distribuzione in cinque classi del numero dei pazienti trattati in 15 giorni.

\begin{tabular}{lc} 
Classi di pazienti trattati in $\mathbf{1 5}$ giorni & Numero di infermeri \\
Da 0 a 5 persone & $357(49 \%)$ \\
\hline Da 6 a 10 persone & $187(26 \%)$ \\
\hline Da 11 a 20 persone & $84(12 \%)$ \\
\hline Da 21 a 50 persone & $80(11 \%)$ \\
\hline Da 51 a 100 persone & $18(2 \%)$ \\
\hline Totale & 726
\end{tabular}

Tabella 1. Provincia della sede di lavoro.

\begin{tabular}{|c|c|c|c|c|c|c|c|c|c|c|c|c|c|c|c|c|c|c|c|}
\hline \multicolumn{20}{|c|}{ Numero intervistati in base alla sede di lavoro delle province italiane } \\
\hline $\mathrm{AL}$ & 1 & $\mathrm{BN}$ & 3 & $\mathrm{CH}$ & 2 & FC & 1 & IS & 1 & $\mathrm{MO}$ & 7 & PG & 2 & RE & 6 & TO & 11 & VE & 9 \\
\hline $\mathrm{AN}$ & 6 & $\mathrm{BO}$ & 4 & $\mathrm{CL}$ & 3 & $\mathrm{FG}$ & 7 & LC & 1 & MT & 1 & PI & 3 & $\mathrm{RM}$ & 446 & $\mathrm{TP}$ & 2 & VI & 10 \\
\hline $\mathrm{AO}$ & 2 & BR & 1 & $\mathrm{CN}$ & 4 & FI & 2 & LE & 2 & NA & 5 & PR & 1 & $\mathrm{RN}$ & 12 & TR & 3 & VR & 6 \\
\hline $\mathrm{AP}$ & 3 & BS & 1 & $\mathrm{CO}$ & 1 & FM & 4 & LI & 1 & NO & 1 & PU & 1 & $\mathrm{RO}$ & 3 & TS & 1 & VT & 11 \\
\hline $\mathrm{AR}$ & 1 & BT & 2 & CR & 1 & FR & 3 & LT & 19 & $\mathrm{NU}$ & 2 & PV & 1 & $\mathrm{SA}$ & 3 & TV & 1 & & \\
\hline $\mathrm{AT}$ & 2 & BZ & 2 & $\mathrm{CS}$ & 3 & GE & 5 & LU & 3 & OG & 2 & $\mathrm{PZ}$ & 1 & SI & 1 & UD & 3 & & \\
\hline BA & 11 & $\mathrm{CB}$ & 5 & CT & 4 & GR & 1 & $\mathrm{MC}$ & 4 & PA & 7 & $\mathrm{RA}$ & 4 & $\mathrm{TA}$ & 6 & VA & 1 & & \\
\hline BI & 3 & $\mathrm{CE}$ & 1 & $\mathrm{CZ}$ & 2 & IM & 2 & MI & 16 & PD & 5 & $\mathrm{RC}$ & 6 & $\mathrm{TE}$ & 1 & $\mathrm{VC}$ & 1 & & \\
\hline
\end{tabular}


dev'essere valutato in modo globale. ${ }^{7,8}$ Non utilizzare sistemi di valutazione oggettiva validati conduce a una pianificazione impropria degli interventi. Le domande proposte nel questionario si riferiscono a tre aspetti: i) la valutazione del rischio; ii) la valutazione della lesione; iii) la valutazione nutrizionale.

Per offrire riscontro della reale valutazione corretta dei tre parametri, a chi ha dato risposta positiva si è chiesto di specificare lo strumento utilizzato. L'inserimento di uno strumento incongruo costituisce prova della mancata conoscenza e utilizzo di tale strumento.

Lo studio non prende in considerazione la valutazione del dolore, ${ }^{11}$ in quanto obbligo di legge. ${ }^{12}$ In letteratura merita la predisposizione di indagini finalizzate: al riguardo si rimanda ai siti dedicati ${ }^{13,14} \mathrm{e}$ a documenti di posizionamento.

\section{La valutazione del rischio}

Nella domanda non è stato specificato un aspetto del rischio per non indurre all'unilateralità della risposta $\mathrm{e}$ per poter determinare quali sono i rischi maggiormente valutati. Alla domanda hanno dichiarato di valutare rispettivamente (Figura 4C): i) 34,85\% Sİ; ii) 65,15\% NO. Le scale più utilizzate (Figura 4D) tra le risposte positive sono Braden 71\% (n. 182) e Norton 20\% (n. 53). Solo il $12 \%$ ha indicato anche la scala Conley di valutazione del rischio di caduta. Ha indicato una scala non idonea per la valutazione del rischio il 3\% di coloro che valutano. La percentuale che realmente valuta il rischio si attesta al 30,8\%: preoccupante evidenza di scarsa sensibilità alla prevenzione.

\section{Valutazione della lesione}

Solo il 22,04\% dichiara di valutarla e il 77,96\% di non valutarla (Figura 5A). A coloro che rispondono positivamente (n. 160 professionisti) è stato richiesto di specificare la scelta dello strumento: n. 22 utilizzano la descrizione dimensionale, n. 13 utilizzano il TIME, n. 2 scelgono il Push Tool, seguono altri sistemi anche di altre categorie. La risposta, lasciata volutamente libera, è stata disomogenea e non congrua.

\section{EZIOLOGIA DI LESIONI TRATTATE}

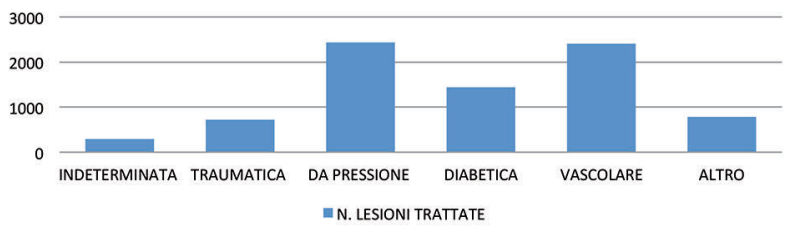

Figura 3. Frequenza delle lesioni trattate secondo eziologia.

\section{A) USO DI DOCUMENTAZIONE STANDARDIZZATA}

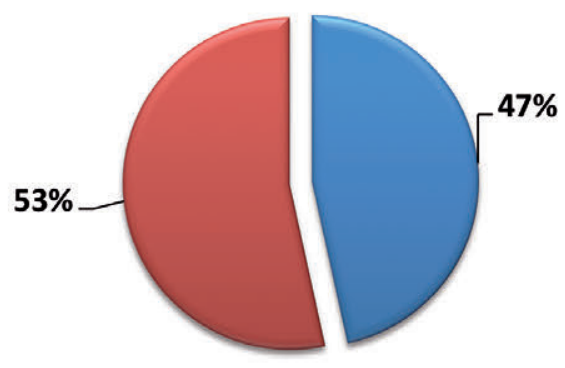
TIPO DI DOCUMENTAZIONE
UTILIZZATA

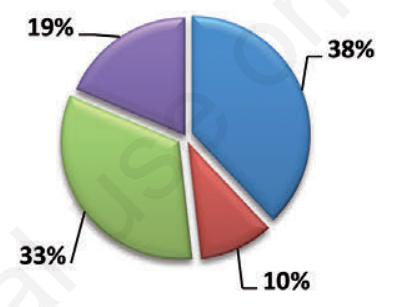

$\square$ CARTELLA

$\square$ APPUNTI

PERSONALI

$\triangle D I A R I O D I$

CONSEGNA

C)

VALUTAZIONE DEL RISCHIO

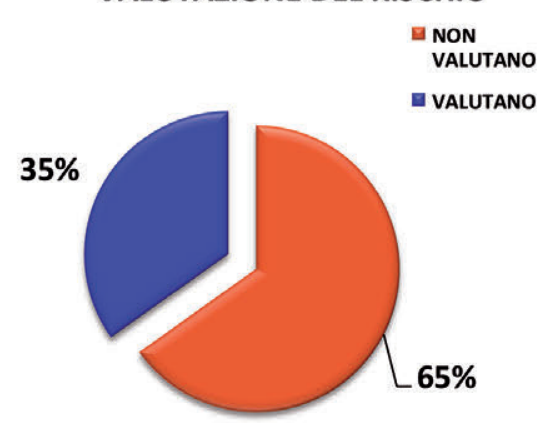

D) VALUTAZIONE DEL RISCHIO

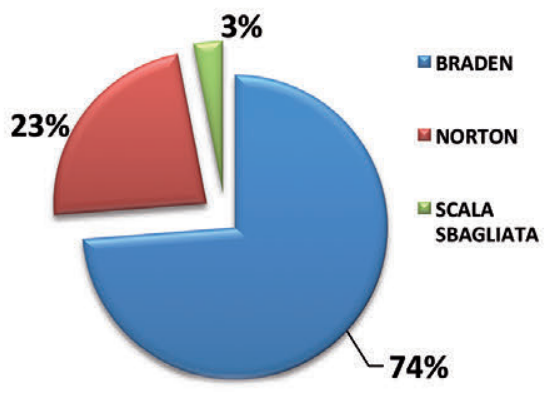

Figura 4. A) Uso di documentazione standardizzata; B) Tipo di documentazione; C) Valuta il rischio? D) Scale prevalentemente utilizzate per la valutazione del rischio di lesione. 


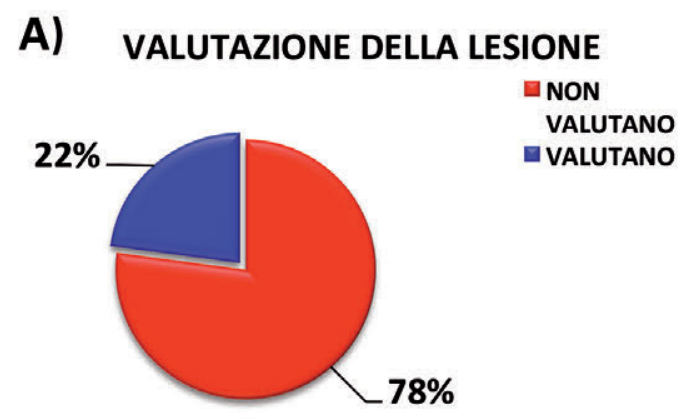

B) VALUTAZIONE NUTRIZIONALE

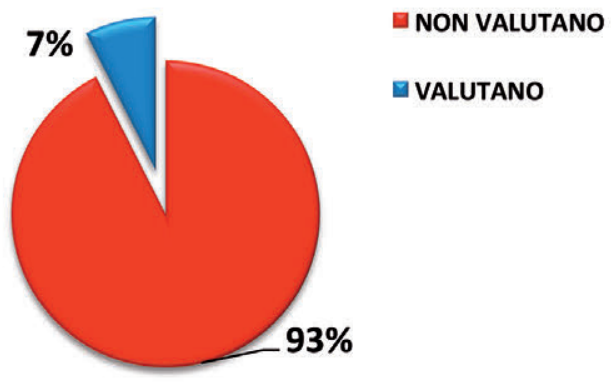

\section{C) STRUMENTO GUIDA}

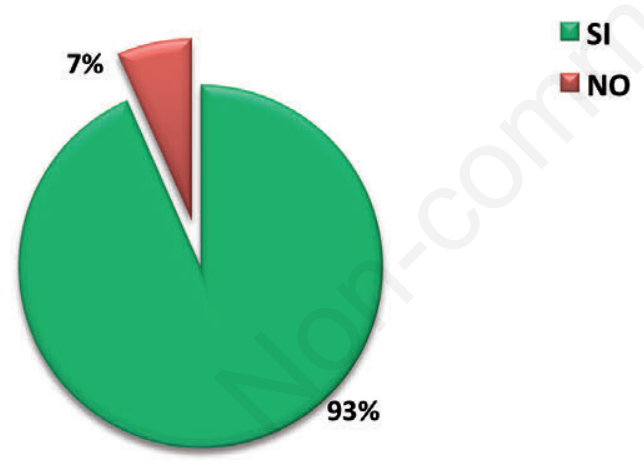

Figura 5. A) Valuta la lesione?; B) Esegue la valutazione nutrizionale?; C) Necessità di avere uno strumento guida.

\section{Valutazione dello stato/rischio nutrizionale}

Quasi la totalità del campione (93\%) non effettua la valutazione del rischio nutrizionale. Fra le n. 57 risposte positive le scale utilizzate risultano essere MNA n. 22 (40\%) e MUST n. 11 (19\%) (Figura 5B). La valutazione nutrizionale è la meno eseguita. Alcuni professionisti hanno indicato come strumento di valutazione il BMI (n. 20), o la scala di Braden (n. 33): da ciò emerge un'importante carenza formativa.

\section{Necessità di avere uno strumento che guidi la scelta degli interventi}

Alla domanda: Sente l'esigenza di avere uno strumento che guidi e documenti i suoi interventi a domicilio come la cartella infermieristica? hanno risposto (Figura 5C): i) $93,39 \%$ Sİ; ii) $6,61 \%$ NO.

\section{Approfondimento delle risposte negative}

Vista l'alta percentuale $(58,38 \%$ sul totale) delle persone che non valutano alcun parametro proposto né documentano la propria attività, sono stati analizzati i dati per individuare un generico profilo ed eventualmente il target d'indirizzo formativo (Tabella 3): per quanto attiene il profilo, indicativamente identifica il professionista con più di 40 anni e con più di 5 anni di servizio, con scarsa/media attività di medicazione e che, abitualmente, non documenta il proprio operato. Tale dato è però banalmente inficiato dalla composizione del campione, che vede il $72 \%$ con più di 5 anni di servizio e il $54 \%$ di età superiore ai 40 anni.

\section{Management e percorso del paziente}

Si è scelto di indagare chi stabilisca il trattamento delle lesioni e la conoscenza di servizi wound care-dedicati. $\mathrm{Nel}$ $37 \%$ dei casi è il medico, nel 63\% 1'infermiere (Figura 6A).

\section{Informazione dell'esistenza dei servizi dedicati alla cura delle lesioni cutanee}

Ha risposto positivamente alla domanda il $41 \%$ del campione, negativamente il 59\% (Figura 6B).

Tabella 3. Analisi comparativa delle risposte negative all'utilizzo di strumenti evidence best practice (EPB).

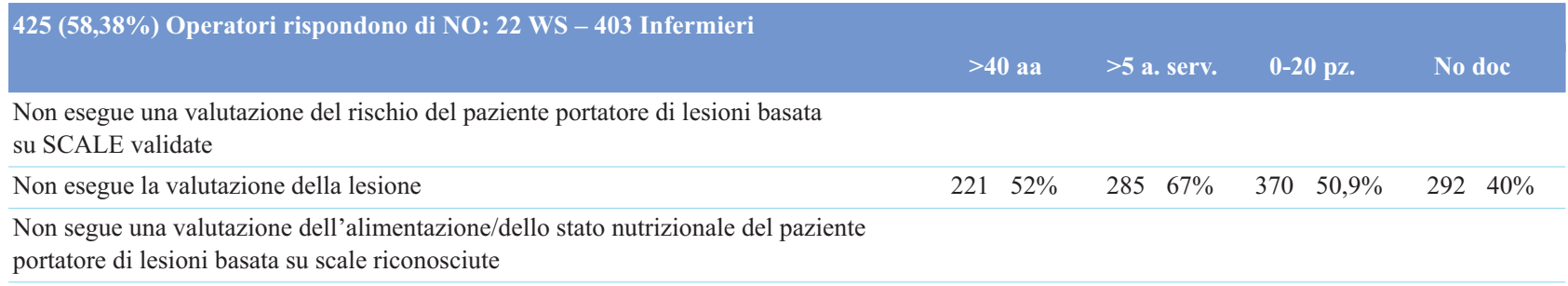




\section{Informazione/conoscenza della formazione post-base dedicata}

La maggior parte degli infermieri $(70 \%)$ dichiara di conoscere l'esistenza della formazione post-base universitaria fornita dal Master di I livello (Figura 6C).

A) CHI STABILISCE IL TRATTAMENTO
DELLA LESIONE

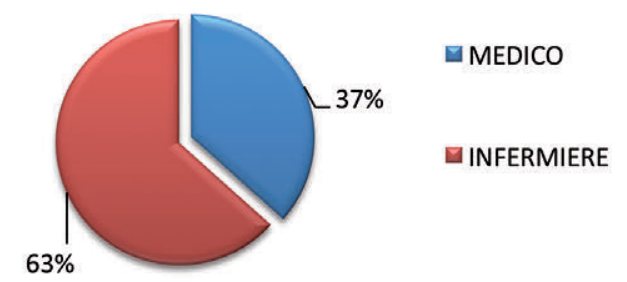

\section{B) È al corrente dell'esistenza di servizi wound care dedicati ?}

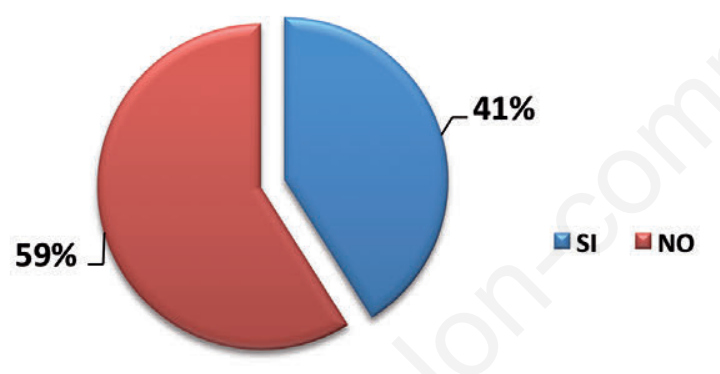

C)

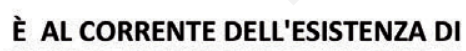 MASTER UNIVERSITARI DEDICATI AL WOUND CARE?}

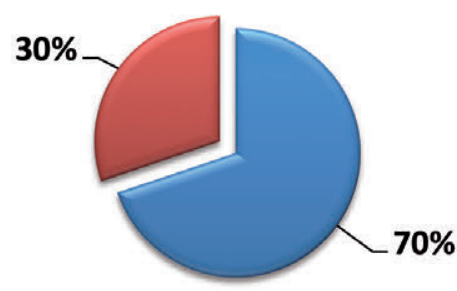

$\square$ SI $\square$ NO

Figura 6. A) Figura professionale che stabilisce la cura della lesione (percentuale); B) Risposte alla domanda: È al corrente dell'esistenza di servizi wound care dedicati? (percentuale); C) Risposte alla domanda: È al corrente dell'esistenza di corsi di Master di I livello dedicati al wound care? (percentuale).

\section{Analisi comparativa dei dati}

\section{Distribuzione delle risposte positive (726 infermieri)}

La percentuale maggiore di risposte positive (Figura 7) riguarda le ultime tre domande: i) l'esigenza di uno strumento dedicato che guidi la scelta del trattamento; ii) la conoscenza dei servizi dedicati al wound care; iii) la conoscenza della formazione universitaria del Master di I livello. Si è voluto indagare sul comportamento operativo delle persone che hanno dimostrato di avere una particolare sensibilità rispetto al problema formativo: l'andamento è proporzionalmente invariato rispetto al totale della popolazione.

\section{Confronto modus operandi struttura - struttura/territorio}

Per meglio focalizzare le scelte operative, se condizionate o meno dall'ambiente di lavoro, sono state confrontate 2 popolazioni, selezionate in base ai modi di risposta (Figura 8): i) solo in struttura (in numero maggiore); ii) in struttura e territorio; iii) solo territorio.

Sono stati selezionati gli operatori che seguono un numero ampio di pazienti ( $>20$ in $15 \mathrm{gg}$.), considerando il numero di pazienti l'indicatore di servizio specialistico, ipotizzando che l'esigenza di lavorare costantemente su pazienti con lesioni costituisca incentivo a formarsi e a

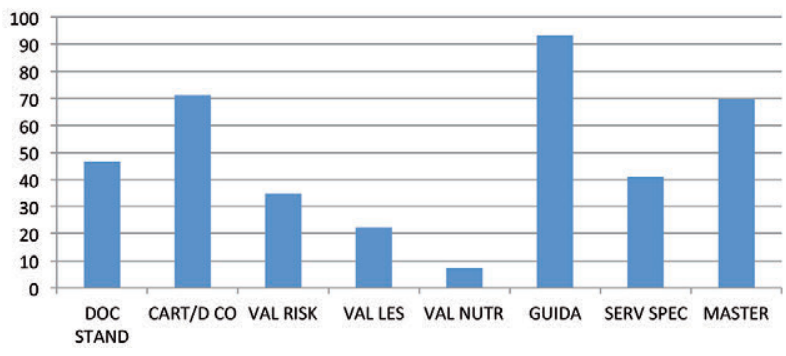

Figura 7. Distribuzione per modalità delle risposte positive.

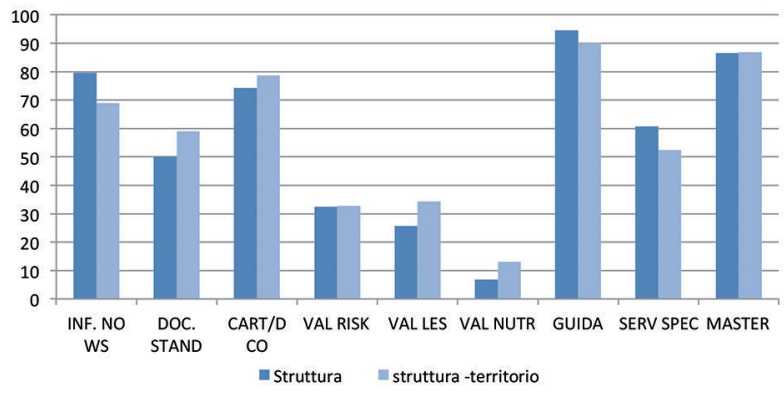

Figura 8. Distribuzione percentuale delle popolazioni di infermieri che trattano da 20 a 100 pazienti ogni 15 giorni, che lavorano in struttura e infermieri che lavorano sul territorio-struttura a confronto. 
conoscere strumenti validati e procedure appropriate al trattamento (Figura 9).

Circa la metà degli infermieri (49\%) segue un numero esiguo di pazienti nell'arco di $15 \mathrm{gg}$. (0-5), e 11\% segue 21-100 persone con lesioni. All'analisi (Tabella 4) il modus operandi di coloro che seguono più di 20 pazienti, risultano essere in maggioranza generalisti, con andamento simile tra diversi ambienti di lavoro.

Non vi sono differenze di distribuzione delle risposte positive riguardo la valutazione del rischio tra un campione e l'altro; un leggero miglioramento si nota tra quelli che svolgono attività anche sul territorio, con riferimento alla valutazione della lesione (25\% solo struttura vs 34\% struttura/territorio) e alla valutazione nutrizionale $(6 \%$ solo struttura $v s$ 13\% struttura e territorio).

L'analisi dei dati rileva che il $74 \%$ degli operatori (n. 101 su n. 136) non ha una formazione specifica. L'uso di una documentazione standardizzata (media 53\%) cartelladiario è scarsa; sul totale n. 13 operatori su n. 135 eseguono la valutazione nutrizionale; si trascura il fatto che

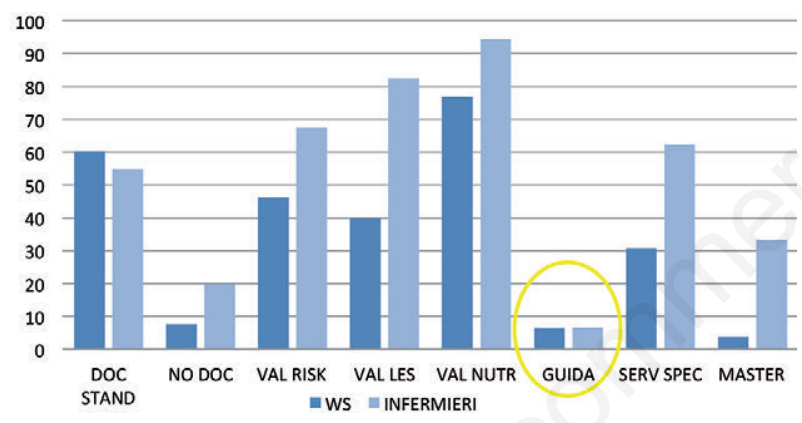

Figura 9. Confronto wound specialists-infermieri. Distribuzione riposte negative (dato percentuale relativo). la documentazione della cura di una lesione è parte integrante della cartella clinica. ${ }^{15}$ Una piccola parte degli infermieri sceglie di non documentare il proprio operato se non altro in un documento non istituzionale (Tabella 4).

In entrambi i casi più del $90 \%$ dichiara di volere uno strumento che lo aiuti nella scelta dell'intervento nella pratica clinica: se ne può dedurre che, anche dove presente una documentazione standardizzata, essa non risponde alle esigenze del wound care.

L'unico valore che si distacca dalla distribuzione è relativo alla penultima domanda: gli infermieri che lavorano in struttura hanno una conoscenza maggiore dell'esistenza dei servizi dedicati alla cura delle lesioni. Ciò può trovare motivazione nel fatto che tali centri, ove esistenti, sono in maggior misura inseriti in strutture ospedaliere e molto meno rappresentati nel territorio.

\section{Confronto tra wound specialists e infermieri}

\section{Analisi risposte negative}

Si rende necessario comprendere se i comportamenti a rischio dei professionisti sono dovuti al livello di formazione professionale raggiunta: sono state analizzate le risposte negative secondo la formazione dichiarata, confrontando le scelte operate dai WS con quelle del rimanente campione (Figura 9).

L'attività dei WS risulta leggermente migliore; circa metà $(46 \%)$ dichiara di non effettuare la valutazione del rischio e il $76,9 \%$ non esegue la valutazione nutrizionale della persona che cura: dato allarmante. I WS valutano maggiormente la lesione rispetto agli operatori senza una formazione specifica, ma $39,7 \%$ di loro (rispetto a $82,5 \%$ infermieri) non descrivono la lesione secondo parametri oggettivabili riconosciuti dalla comunità scientifica; un

Tabella 4. Confronto su uso best practice tra operatori rispetto al posto di lavoro.

\begin{tabular}{|c|c|c|c|c|c|}
\hline Totale 135 operatori $\geq 20-100$ pazienti ogni 15 giorni & & & & & \\
\hline Posto di lavoro & Solo p & truttura & $\begin{array}{r}\text { Struttu } \\
\text { Sold }\end{array}$ & $\begin{array}{l}\text { erritorio - } \\
\text { ritorio }\end{array}$ & Totale \\
\hline Operatori & 74 & $54 \%$ & 61 & $45 \%$ & 135 \\
\hline Di cui infermieri non specializzati & 59 & $79 \%$ & 42 & $68,8 \%$ & 101 \\
\hline Utilizza una documentazione standardizzata & 37 & $50 \%$ & 36 & $59 \%$ & 73 \\
\hline Utilizza cartella e diario di consegna & 55 & $74,3 \%$ & 48 & $78 \%$ & 104 \\
\hline Esegue una valutazione del rischio basata su SCALE validate & 24 & $32 \%$ & 20 & $32,7 \%$ & 44 \\
\hline Esegue la valutazione della lesione con metodi idonei & 19 & $25 \%$ & 21 & $34 \%$ & 40 \\
\hline $\begin{array}{l}\text { Esegue una valutazione dell'alimentazione/dello stato nutrizionale basata su } \\
\text { SCALE riconosciute }\end{array}$ & 5 & $6,7 \%$ & 8 & $13 \%$ & 13 \\
\hline Vorrebbe avere uno strumento dedicato che guidi e documenti i suoi interventi & 70 & $94 \%$ & 55 & $90 \%$ & 125 \\
\hline $\begin{array}{l}\text { È al corrente dell'esistenza di servizi wound care dedicati (per la cura ed il trattamento } \\
\text { delle lesioni cutanee) nella sua zona }\end{array}$ & 45 & $60 \%$ & 32 & $52 \%$ & 77 \\
\hline È al corrente dell'esistenza di master universitari di primo livello dedicati al wound care & 64 & $86 \%$ & 62 & $100 \%$ & 117 \\
\hline
\end{tabular}


dato singolarmente discordante è che $\mathrm{n} 3$ di loro dichiarano di non conoscere l'esistenza del percorso universitario di formazione, mostrandosi apparentemente inconsapevoli del livello di formazione conseguito. Gli infermieri senza una formazione specifica non utilizzano documentazione standardizzata in una percentuale del $54,9 \%$, la valutazione della lesione non viene eseguita da $82,5 \%$, e il $94 \%$ non valuta lo stato nutrizionale del paziente.

\section{Analisi del campione laziale}

Considerato che la maggioranza del campione è rappresentata da infermieri operanti nel Lazio n. 479 $(65,79 \%)$, si è analizzato il loro profilo (Figura 10), che risulta essere: i) lievemente più giovani della totalità degli infermieri; ii) solo il 6\% WS; iii) circa il 67\% lavora solo in struttura; iv) altrettanti da più di 5 anni.

Circa il 60\% degli infermieri laziali non effettua o effettua medicazioni a meno di 5 pazienti in 15 giorni. Più del 50\% degli infermieri laziali non dispone di documentazione standardizzata, poco più di $1 / 4$ utilizza propri appunti o non documenta gli interventi effettuati sul paziente per la cura della lesione. Il 17\% risponde di effettuare la valutazione della lesione non conoscendone gli strumenti EBP, e meno della metà dei rispondenti positivamente danno corrette risposte. Il 17\% dichiara di effettuare la valutazione del rischio/stato nutrizionale, ma n. 13 persone non utilizzano lo strumento appropriato. Quasi tutti gli infermieri del Lazio vorrebbero uno strumento che sostenga la loro attività per la cura del paziente portatore di lesione, ma ad oggi il $40 \%$ dei piani di trattamento sono decisi dal medico.

Il 35\% del campione di riferimento conosce un servizio di wound care, e più della metà conosce il Master di I livello in wound care. Il quadro, con differenze più o meno significative, si ripete sia a livello della regione Lazio, sia nel resto delle realtà locali rilevate.

\section{DISCUSSIONE}

La ricerca aperta condiziona i dati in funzione degli eventi e della distribuzione e accessibilità alla partecipazione alla ricerca. La validazione del questionario secondo la Formula Kuder e Richardson (0-1): i) alla prima somministrazione al $\mathrm{t}^{0}$ risulta coerenza interna alta, pari a $\mathrm{P}=0,704177323$; ii) alla seconda somministrazione $\mathrm{t}^{1} \mathrm{è}$ pari a $\mathrm{P}=0,647368421$ comunque accettabile.

\section{Profilo della popolazione coinvolta}

Dall'indagine risulta che la maggior parte della popolazione degli infermieri coinvolta nell'indagine è di genere femminile (71\%), il 54\% di età superiore ai 40 anni. La maggioranza (84\%) è infermiere generalista che prevalentemente $(63 \%)$ esercita la professione in struttura ospedaliera, non in contraddizione con i dati demografici della popolazione infermieristica iscritta al Collegio IPA-

\section{LAZIO: ETA'}

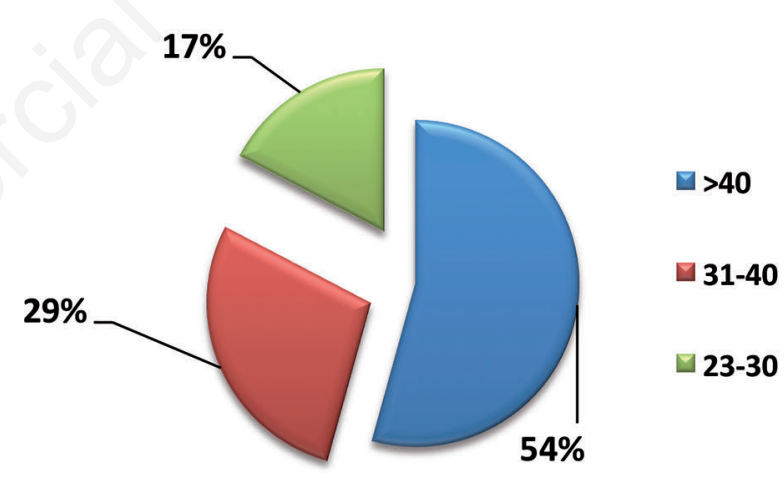

Figura 10. Distribuzione per età del campione laziale.

Tabella 5. Tutta la popolazione intervistata: wound specialists vs infermieri che non scelgono di operare secondo evidence best practice (EPB).

\begin{tabular}{|c|c|c|c|c|}
\hline 78 WS us 648 infermieri & WS 78 & $\%$ & INF 648 & $\%$ \\
\hline Non utilizza una documentazione standardizzata & 47 & 60,2 & 356 & 54,9 \\
\hline Non documenta i suoi interventi su pazienti portatori di lesioni (appunti personali/non documento) & 8 & 7,7 & 129 & 19,9 \\
\hline Non esegue una valutazione del rischio del paziente portatore di lesioni basata su scale validate & 36 & 46,1 & 437 & 67,4 \\
\hline Non esegue la valutazione della lesione & 31 & 39,7 & 535 & 82,5 \\
\hline $\begin{array}{l}\text { Non esegue una valutazione dell'alimentazione/dello stato nutrizionale del paziente portatore di lesioni } \\
\text { basata su scale riconosciute }\end{array}$ & 60 & 76,9 & 612 & 94,4 \\
\hline $\begin{array}{l}\text { Non vorrebbe avere uno strumento dedicato che guidi e documenti i suoi interventi su pazienti portatori } \\
\text { di lesioni }\end{array}$ & 5 & 6,4 & 43 & 6,6 \\
\hline $\begin{array}{l}\text { Non è al corrente dell'esistenza di servizi wound care dedicati (per la cura ed il trattamento delle lesioni } \\
\text { cutanee) nella sua zona }\end{array}$ & 24 & 30,7 & 404 & 62,3 \\
\hline Non è al corrente dell'esistenza di master universitari di I livello dedicati al wound care & 3 & 3,8 & 216 & 33,3 \\
\hline
\end{tabular}


SVI in attività lavorativa negli ultimi anni, con una forchetta del 10-20\% ca., nelle diverse realtà regionali.

L'ultimo tasso registrato d'ingresso degli studenti al corso di laurea di I livello in Infermieristica è del 37\%, percentuale molto inferiore alla maggior parte dei Paesi OCSE. ${ }^{10}$ I giovani laureati in Infermieristica, tra i 25 e i 34 anni, non trovano facilmente lavoro e registrano un tasso di occupazione del $62 \%$ contro una media OCSE dell' $83 \%$.

In Italia gli infermieri sono n. 6 (6:1000 abitanti) ${ }^{16}$ ivi compresi quelli non a diretto contatto con il pubblico. Coloro che hanno conseguito il titolo di WS (Master di I livello in Wound Care) costituiscono 1'11\% della popolazione analizzata; il dato sostanzialmente riflette lo stato generale in quanto gli operatori con specializzazione universitaria post-laurea sono generalmente pochi. Al momento non abbiamo a disposizione dati nazionali dell'entità degli infermieri Specialist. L'acquisizione di una formazione specialistica è sempre originata da una motivazione individuale piuttosto che da una strategia organizzativa: nella realtà laziale sono sorti negli ultimi decenni centri dedicati di difficile mappatura, piuttosto di centri di cura sia pubblici sia privati in assenza di un chiaro progetto strategico sanitario regionale sui percorsi di cura delle persone affette o a rischio di lesione.

La sede di lavoro è un indicatore dei poli di attrazione professionale: il 63\% lavora solo in struttura, e, fortunatamente, le prospettive si allargano per l'incremento in questi ultimi anni dell'attività ambulatoriale-territoriale. Il dato è viziato non solo dal contesto, ma anche dal fatto che la motivazione a partecipare ai congressi di vulnologia in occasione dei quali le persone sono state reclutate nell'indagine, non sempre era ed è l'interesse professionale e/o l'impegno lavorativo nel settore, ma anche l'esigenza di soddisfare il debito formativo ECM, ${ }^{9}$ e che i giovani che lavorano con contratto a termine o come liberi professionisti spesso non hanno la possibilità economica di partecipare agli eventi. Allo scopo di rilevare l'esperienza lavorativa nel campo del wound care l'attenzione si è concentrata su due variabili: anzianità di lavoro e numero di pazienti trattati in 15 giorni. Il 72\% del campione ha più di 5 anni di servizio, dato coerente con l'età maggiormente dichiarata. Il 13\% degli infermieri segue costantemente da n. 21 a n. 100 pazienti ogni 15 giorni, n. 50 le medicazioni effettuate mediamente in 15 giorni; considerando il costo economico per ogni medicazione, gli effetti sulla vita del paziente/famiglia in termini di costo-salute, costo-tempo, tempo-lavoro, il bilancio di tale attività è inversamente proporzionale all'efficacia dei trattamenti effettuati.

L'eziologia della lesione trattata rispecchia il dato generale con la prevalenza delle lesioni da pressione e di quelle vascolari. È rilevante notare che tra tutte le lesioni trattate solo il 3\% ha eziologia indeterminata, con proba- bilità che si verifichi un trattamento inappropriato. È un margine di errore troppo alto nella clinica: un solo trattamento incongruo potrebbe causare un danno tale da annullare i vantaggi ottenuti da giorni o mesi precedenti di lavoro. ${ }^{17,18}$

\section{Strumenti adottati per la valutazione del paziente affetto da lesione}

\section{Documentazione adottata}

Il $47 \%$ non documenta l'attività o non dispone di una documentazione standardizzata. Il 30\% circa sceglie di non documentare (Figura 4B) o di farlo con superficialità, che si svolga l'attività come dipendente sia come libero professionista: si tratta di una criticità da affrontare con urgenza.

È sottovalutato l'obbligo legale ${ }^{19}$ di documentare le prestazioni eseguite (in caso di mancato rispetto v. artt. 476 e 479 C.P.). L'assenza della documentazione su cartelle/fascicoli standardizzati con accesso multidisciplinare influisce in modo negativo sulla qualità della prestazione, venendo a mancare la comunicazione interdisciplinare, l'uniformità di linguaggio e il confronto e il raggiungimento degli obiettivi assistenziali condivisi.

La centralità del paziente nella sua interezza di persona, non limitata alla sola lesione, è condizione imprescindibile per il raggiungimento di una buona gestione. . $^{4-6}$ Uno stato clinico generale alterato non permetterà i processi di riparazione tessutale e la guarigione della lesione: aspetto questo troppo spesso trascurato, in specie, ma non solo, da coloro che sono privi di formazione specialistica in vulnologia. Il rischio, concreto e immediato, è il notevole aumento della spesa sanitaria dedicata alla cura delle lesioni e l'evoluzione verso la cronicizzazione. ${ }^{19,20}$

In questa indagine ci si è concentrati su tre aspetti quali la valutazione del rischio, del rischio nutrizionale e la lettura della lesione: i quesiti sono stati elaborati in modo da non influenzare le risposte o di ridurre al minimo tale fenomeno, ed è stata lasciata completamente libera la risposta sul tipo di metodo adottato, al fine di rilevare incongruenze, poi evidenziate.

$\grave{E}$ tale rilevazione che permette al ricercatore di orientare in modo peculiare la carenza formativa/di aggiornamento del professionista. Fra attività non eseguita e risposte incongrue meno di un terzo della popolazione esegue una corretta valutazione del paziente: particolarmente preoccupante considerando che $72 \%$ del campione ha un'esperienza lavorativa di più di 5 aa., dunque non esordiente, e che $51 \%$ segue più di 5 pazienti nell'arco di $15 \mathrm{gg}$. Benché valido, il dato riflette prevalentemente la situazione nelle regioni più rappresentate, ove maggiormente si ritiene impellente provvedere a formazione e aggiornamento capillari, considerando i contenuti e i percorsi della formazione base. 
Dalle risposte disomogenee ottenute è evidente come non sia chiaro il significato e vi sia confusione tra monitoraggio, valutazione e classificazione delle lesioni, e come l'importanza della valutazione si poco percepita: ma è impensabile portare a guarigione una lesione senza valutarla. ${ }^{21}$ Se le altre valutazioni sono risultate scarse, quella dello stato/rischio nutrizionale appare ancora più grave, in quanto valutata da solo il $7,44 \%$, rispetto al $92,56 \%$ che dichiara di non praticarla. In sintesi il reale stato dell'arte della valutazione paziente/lesione mostra che la valutazione del rischio viene eseguita da $35 \%$ degli intervistati, la valutazione della lesione da $23 \%$ e dello stato/rischio nutrizionale da $7 \%$. Si è scelto in base alla parzialità dei dati raccolti d'individuare il profilo del professionista che sceglie malpractice: indicativamente il professionista con più di 40 aa. con più di 5 aa. di servizio, che ha una bassa/media attività di medicazione e che abitualmente non documenta il suo operato.

La situazione riscontrata si può definire particolarmente grave, incompatibile con un sistema sanitario sempre più, a giusta ragione, orientato a qualità $\mathrm{e}$ appropriatezza a ogni livello. La carenza formativa ostacola i percorsi di diagnosi e di accesso a cure idonee. ${ }^{22,23}$

\section{Bisogno formativo-necessità di avere uno strumento che guidi la scelta degli interventi}

Alla luce degli sconfortanti dati si rileva che in grande maggioranza $(93,3 \%)$ il professionista senta il bisogno di uno strumento che guidi la scelta degli interventi. Più della metà degli infermieri stabiliscono il trattamento $(63 \%)$.

Il 59\% degli intervistati dichiara di conoscere l'esistenza di servizi dedicati. I servizi specialistici dedicati in territorio scarseggiano, e riteniamo che l'alta percentuale di risposte positive sia dovuto al fatto che la maggioranza del campione lavora in struttura sanitaria/ospedaliera ove la presenza degli ambulatori è nota, mentre il $51 \%$ conosce la formazione universitaria post base del Master di I livello in Wound Care; dato incomprensibile: le 3 persone che hanno dichiarato di possedere il titolo che rispondono a quest'ultima domanda negativamente.

\section{CONCLUSIONI}

Distribuzione delle risposte positive di tutti gli intervistati (Figura 7): si è voluto indagare sull'operatività delle persone che hanno dimostrato di avere una particolare sensibilità verso la formazione. La distribuzione delle popolazioni, rispetto alle risposte positive al questionario proposto, presenta le frequenze maggiori in corrispondenza dell'uso di documentazione standard e della cartella sanitaria, con scarso utilizzo della valutazione dell'esposizione del paziente al rischio di lesione, scarsa applicazione di semplici modelli per la descrizione della lesione. È sottovalutata la correlazione tra stato nutrizionale e lesione per lo scarso utilizzo di strumenti validati nella rilevazione del rischio/status nutrizionale. Proporzionalmente rimane invariata la distribuzione rispetto all'inquadramento delle persone affette da lesione.

In merito al confronto modus operandi struttura struttura/territorio, sono state confrontate le popolazioni che lavorano solo in struttura con quelle che lavorano sia in struttura sia in territorio e solo territorio, tra i professionisti che trattano più di 20 pazienti in $15 \mathrm{gg}$. al fine di comprendere se l'ambiente di lavoro influenzi le scelte operative.

Più del 20\% degli infermieri riportano di non utilizzare alcuna documentazione clinica standardizzata, nel territorio, la malpractice aumenta il rischio clinico, già elevato per la rotazione del personale e l'alternanza delle figure coinvolte. La documentazione dell'intervento è garanzia di: i) comunicazione tra professionisti; ii) continuità dei trattamenti; iii) coerenza/sicurezza dei trattamenti; iv) fiducia dell'utente nei trattamenti ricevuti.

La valutazione del rischio, la valutazione della lesione e la valutazione del rischio/stato nutrizionale, sono le pratiche meno utilizzate. Solo $32 \%$ esegue la valutazione del rischio, una media di $29 \%$ valuta la lesione e ancor meno, mediamente il 9,55\% valuta lo stato nutrizionale, cause del prolungato o non idoneo trattamento e dell'insuccesso delle cure dei pazienti in carico ai servizi.

Si può concludere che la mancanza di una documentazione integrata e la scarsa conoscenza/uso di strumenti validati è indice di cattiva qualità dei servizi e dovrebbe costituire input per ogni professionista ad aggiornare le sue conoscenze, e da parte delle strutture a: i) adeguare e aumentare l'uso di documentazione standardizzata; ii) valutare la formazione degli operatori di settore; iii) provvedere al miglioramento dello stato formativo attuale; iv) assumere operatori con formazione specifica e/o formarli adeguatamente.

Un operatore che gestisce casualmente/occasionalmente lesioni probabilmente non ha interesse a utilizzare una scala standardizzata, ma ha sempre l'obbligo di informarsi sull'argomento; coloro invece che hanno a che fare ogni giorno con pazienti portatori di lesioni cutanee non possono esimersi da conoscere e fare uso regolare degli strumenti specifici.

Per quanto riguarda il confronto tra ws e infermieri, in merito all'analisi delle risposte negative, il dato preoccupante che si evince (Tabella 5) è che gli operatori che si definiscono esperti nella gestione della lesione il $60,2 \%$ riferiscono di non utilizzare una documentazione standardizzata, essenzialmente non trasmettono la loro attività, segno di una grave mancanza di comunicazione interprofessionale; i WS inoltre dichiarano di non valutare la lesione su parametri riconosciuti: ciò pone lecitamente degli 
interrogativi sulla qualità della formazione acquisita durante il Master universitario di I livello. La formazione deve essere uniformata e riferita a standard di alta qualità e a tal fine controllata.

Leggendo i dati emersi si ritiene di dover ribadire la particolare gravità della situazione riscontrata, incompatibile con un sistema sanitario basato su criteri di qualità ed appropriatezza. La carenza formativa lede il diritto alla salute e quello all'uniformità di accesso alle cure: appare perentoria la necessità di attuare misure e programmi immediati per colmare tali carenze, di sollecitare le strutture ad assumere nei reparti wound care dedicati personale con comprovata formazione specifica.

Analisi del campione laziale: considerato che il gruppo geograficamente più rappresentato è quello laziale, si è scelto di evidenziarne le caratteristiche. Relativamente più giovane (Figura 9), la metà sceglie di non documentare la propria attività, il $69 \%$ non dispone di documentazione standardizzata, altrettanti non valutano il rischio di insorgenza di lesione e ben 7 persone, pari al 1,5\%, di quelle che dichiarano di farlo, utilizzano scale improprie. La valutazione errata espone il paziente a rischio e l'attività impropria derivante a configurarsi in reato. ${ }^{24}$

I dati rilevati descrivono una realtà molto vicina alla percepita, evidenziando la necessità di strumenti idonei alla formazione per il corretto approccio professionale: ${ }^{25}$ la maggior parte del campione vorrebbe avere uno strumento dedicato che guidi e documenti i suoi interventi su pazienti portatori di lesioni. Molti operatori scelgono di non utilizzare strumenti validati per la lettura della lesione, la valutazione del rischio di lesione e dello status/rischio nutrizionale; le organizzazioni sono carenti nel promuovere e incentivare l'utilizzo di documentazione standardizzata e l'implementazione di EBP.

\section{BIBLIOGRAFIA}

1. Toma E, Veneziano ML. Gestione domiciliare del paziente portatore di lesioni cutanee. Cartella Infermieristica domiciliare. Tesi Master I Livello in Wound Care, Università La Sapienza di Roma. Roma; 2011.

2. Bellingeri A. Il prontuario per la gestione delle lesioni cutanee. $2^{\text {a }}$ ed. Pavia: CdG EDRA LSVR; 2014.

3. National Pressure Ulcer Advisory Panel, European Pressure Ulcer Advisory Panel and Pan Pacific Pressure Injury Alliance. Prevention and treatment of pressure ulcers: quick reference guide. Emily Haesler, ed. Osborne Park, Australia: Cambridge Media; 2014.

4. Harding K. Simplifying venous leg ulcer management: consensus recommendations. Wounds International 2015. Available from: http://www.woundsinternational.com.

5. Gensini GF, Fabbri LM, Fini M, Nozzoli C. La medicina della complessità. Firenze: University Press; 2010. Available from: http://www.fupress.com/Archivio/pdf\%5C4564.pdf. 6. Probst S, Seppänen S, Gethin G, et al. EWMA document: home care-wound care. J Wound Care 2014;23:S1-S44.

7. Le Reste JY, Nabbe P, Manceau B, et al. The European general practice research network presents a comprehensive definition of multimorbidity in family medicine and long-term care, following a systematic review of relevant literature. J Am Med Dir Assoc 2013;14:319.

8. Ministero della Salute. Quaderni del Ministero della Salute n. 23, settembre-ottobre 2013. Criteri di appropriatezza clinica.

9. Normativa: Decreto legislativo 229/99 recante Norme per la razionalizzazione del Servizio Sanitario Nazionale, Art. 16-bis, Formazione continua.

10. OCSE. Education at a Glance/Régards sur l'éducation. 2016

11. Silvestri A, Veneziano ML, Toma E. La valutazione del dolore nel paziente portatore di lesioni cutanee. Tesi Master I Livello Wound Care, Università La Sapienza di Roma. Roma; 2017.

12. Legge 15 marzo 2010, n. 38, G.U., n. 65, 19 marzo 2010.

13. The International Association for the Study of Pain. Available from: http://www.iasp-pain.org. Ultimo accesso: febbraio 2017.

14. American Pain Society. Available from: http://www.americanpainsociety.org. Ultimo accesso: Febbraio 2017.

15. National Pressure Ulcer Advisory Panel, European Pressure Ulcer Advisory Panel, and Pan Pacific Pressure Injury Alliance White Paper. The role of nutrition for pressure ulcer management. Adv Skin Wound Care 2015;28:175-88.

16. Fortunato E. Gli infermieri in Italia: quanti sono, che età hanno e come sono distribuiti. L'infermiere 2013;57. Disponibile su: http://www.ipasvi.it/ecm/rivista-linfermiere/rivista-linfermiere-page-16.htm.

17. Documento di Posizionamento AIUC sulla medicazione dell'ulcera cutanea. Acta Vulnol 2014;12:229-34.

18. University of Leicester. Audit Protocol. The nursing management of patients with venous leg ulcers. Clinical practice guideline RCN2006. Available from: http://www.ren.org. uk/publications/pdf/guidelines/venous_leg_ulcers.pdf

19. Schultz GS, Sibbald GR, Falanga V, et al. Wound bed preparation: a systematic approach to wound management. Wound Repair Regen 2003;11:1-28.

20. European Wound Management Association (EWMA). Position document: wound bed preparation in practice. London: MEP Ltd; 2004.

21. Campitiello F, Lauriello C. Percorsi diagnostico-terapeutici ospedale-territorio per la gestione delle ulcere cutanee. AIUC, 2011.

22. Alesse P, Fornaciari M, Palombi M, et al. Progetto di assistenza integrata ospedale-territorio per i pazienti affetti da ulcere cutanee vascolari, da piede diabetico e dalle lesioni cutanee in generale che non tendono ad una facile guarigione. AIUC, 2006.

23. Legge 8 marzo 2017, n. 24. Sicurezza delle cure in sanità, Legge Gelli. Gazzetta Ufficiale 17 marzo 2017.

24. Valderas JM, et al. Defining comorbidity: implications for understanding health and health services. Ann Fam Med 2010;7:357. 